\title{
Current perspectives on Internet-delivered cognitive behavioral therapy for adults with anxiety and related disorders
}

This article was published in the following Dove Press journal:

Psychology Research and Behavior Management

30 January 2014

Number of times this article has been viewed

\section{Louise Mewton \\ Jessica Smith \\ Pieter Rossouw \\ Gavin Andrews}

Clinical Research Unit for Anxiety and Depression, St Vincent's Hospital, Sydney, NSW, Australia
Correspondence: Louise Mewton Clinical Research Unit for Anxiety and Depression, Level 4, O’Brien Centre, St Vincent's Hospital, 394-404 Victoria Street, Darlinghurst, NSW, Australia Tel +6I 28382 |437

Email louisem@unsw.edu.au
Abstract: The aim of the current review is to provide a summary of research into Internet-delivered cognitive behavioral therapy (iCBT) for anxiety disorders. We include 37 randomized controlled trials that examined the efficacy of iCBT programs in adults (aged over 18 years), as compared with waiting list or active control. The included studies were identified from Medline searches and from reference lists, and only published data were included. Several trials of iCBT for generalized anxiety disorder, panic disorder, and social phobia were identified. Two trials of iCBT for obsessive-compulsive disorder were identified, whilst one trial each was identified for hypochondriasis, specific phobia (spiders), and post-traumatic stress disorder. Finally, there were five trials that focused on transdiagnostic therapy for either a range of comorbid anxiety disorders or comorbid anxiety and depression. Between-group effect sizes were moderate to large for all disorders, and ranged from 0.30 to 2.53. iCBT was found to be commensurate with face-to-face cognitive behavioral therapy whether delivered individually or in group format. Guidance may not be necessary for iCBT to be effective for immediate gains, but may be more important in longer-term maintenance of symptom improvement and maximizing patient adherence. The clinical experience of the individual providing guidance does not appear to impact treatment outcomes. Future research needs to focus on the optimal level of guidance required to generate maximum patient benefits, whilst balancing the efficient use of clinician time and resources. Evidence-based contraindications to iCBT should also be developed so that the choice of treatment modality accurately reflects patients' needs. Further research should be conducted into the effective elements of iCBT, as well as the extent to which therapy enhancers and advancing technology can be accommodated into established iCBT frameworks.

Keywords: Internet-delivered cognitive behavioral therapy, iCBT, anxiety disorders

\section{Introduction}

Over a quarter of the Australian population has experienced an anxiety disorder in their lifetime, ${ }^{1}$ but less than $40 \%$ of individuals affected by these disorders have sought treatment for their symptoms. ${ }^{2}$ The most commonly occurring anxiety disorders include generalized anxiety disorder, panic disorder, specific phobia, social phobia (with or without agoraphobia), post-traumatic stress disorder, obsessive-compulsive disorder, and illness anxiety disorder. These disorders are marked by excessive fear, anxiety, and associated avoidance behaviors, but are distinguished from each other by the types of objects or situations that induce fear or avoidance. Anxiety disorders are marked by persistence, rather than transient fear or anxiety, and tend to have their onset in childhood or adolescence. ${ }^{3}$ 
Generalized anxiety disorder is characterized by at least 6 months of prominent tension, worry, and feelings of apprehension surrounding everyday events and problems coupled with symptoms associated with autonomic arousal. ${ }^{4}$ Panic disorder (with and without agoraphobia) is a debilitating condition characterized by recurrent, unexpected panic attacks accompanied by persistent concern about future attacks and possible avoidance of situations and places in which attacks are expected to occur. ${ }^{5}$ Specific phobias are marked by excessive fear or anxiety about a specific object or situation (such as spiders or flying), accompanied by active avoidance of the phobic object or situation. ${ }^{3}$ Social phobia (with and without agoraphobia) is characterized by a persistent and debilitating fear of social situations in which the individual may be exposed to scrutiny by others. ${ }^{6}$ Post-traumatic stress disorder, which is classified as a trauma- and stressor-related disorder in the fifth edition of the Diagnostic and Statistical Manual of Mental Disorders (DSM-5), is characterized by intrusion symptoms and avoidance behaviors following exposure to a traumatic event. ${ }^{3}$ Obsessive-compulsive disorder, classified under the obsessive-compulsive and related disorders in DSM-5, is defined by unwanted and intrusive obsessions and/or compulsions aimed at preventing or reducing anxiety or distress, or averting feared events or situations. ${ }^{3}$ Finally, illness anxiety disorder, or hypochondriasis according to DSM-IV, is classified as a somatoform disorder in both DSM-IV and DSM-5, and refers to excessive concern over health concerns and associated reassurance-seeking from health professionals. ${ }^{3,7}$

Pharmacotherapy (selective serotonin reuptake inhibitors) and cognitive behavioral therapy (CBT) are both effective in the treatment of anxiety disorders. ${ }^{8,9}$ Overall, pharmacotherapy and CBT are equivalent in terms of treatment outcomes, but adherence to CBT appears to be higher, ${ }^{10}$ possibly due to the unwanted side effects of pharmacotherapy. As a result, CBT has emerged as the initial treatment of choice for patients with anxiety disorders. ${ }^{11} \mathrm{CBT}$ is an umbrella term for various treatments that focus on challenging cognitive biases (through cognitive restructuring) and behaviors (ie, through graded exposure and relaxation training) that perpetuate the anxiety cycle. ${ }^{12}$ Whilst there are various other psychological treatments available for anxiety disorders, CBT has the strongest supporting evidence. ${ }^{13}$

Despite the effectiveness of face-to-face CBT, numerous barriers to treatment exist, including the direct and indirect costs of treatment, the limited availability of mental health professionals, stigma, and the difficulty of patients attending treatment during office hours. ${ }^{14}$ Alternatives to face-to-face therapy have been developed that have the potential to overcome some of these barriers, and several innovative approaches for improving access to evidence-based care have been proposed..$^{15}$ One possible solution is to develop less therapist-intensive treatments that can be delivered from a distance. The growth of fast and reliable Internet services offers new and exciting treatment opportunities, with Internet-delivered CBT (iCBT) showing considerable promise in the effective treatment of a range of mental disorders. ${ }^{15}$ iCBT has been developed as a way of providing accessible psychotherapy without the need for intensive therapist involvement. Typically, the patient follows a structured and interactive treatment program available on the Internet in conjunction with limited therapist contact via telephone calls, texts, or emails. ${ }^{16}$ Content is usually based on a predetermined syllabus that is presented in a series of lessons, and accompanied by homework assignments and supplementary information. Existing iCBT programs are almost exclusively grounded in evidence-based approaches ${ }^{17}$ and aim to cultivate new types of behavior and thinking patterns in a standard cognitive-behavioral framework. ${ }^{18}$

iCBT has distinct advantages over traditional face-toface CBT for both clients and health care professionals. ${ }^{18}$ Successful distribution of CBT treatment programs can be severely hampered by the lack of trained CBT therapists ${ }^{19}$ and access to evidence-based treatment is limited by a wide variety of factors, including long patient waiting lists, stigma, and patient cost. ${ }^{20}$ Internet-based distance psychotherapies may be of particular value for people living in remote areas, for those with irregular or inconvenient work schedules, and for individuals who cannot afford or fear face-to-face therapy due to the nature of their anxiety disorder. ${ }^{21}$ Internet-delivered treatments are highly scalable and able to meet the growing demand for psychiatric care. ${ }^{22}$ iCBT protocols are also highly structured, ensuring standardized delivery of treatment components and the minimization of therapist drift. ${ }^{23}$ Finally, the ability to embed questionnaires within iCBT programs allows for automatic reports of patient progress throughout the treatment process and an accurate record of patient adherence, enhancing the speed and ease with which the efficacy of the treatment can be established. ${ }^{24}$

\section{Current review}

The number of studies examining the efficacy of Internet interventions for anxiety disorders has grown rapidly. As a result, several recent systematic reviews and meta-analyses have been conducted..$^{15,18,24-30}$ The main aim of the current review is to focus on issues outside the scope of these 
previous reviews and meta-analyses, which have tended to focus on the evidence for efficacy. Only randomized controlled trials of Internet-delivered CBT (including psychoeducation, applied relaxation, and graded exposure) for a range of anxiety and related disorders were considered for inclusion. Studies limited to individuals under the age of 18 years were excluded. Trials of computerized CBT, where the patient is required to visit a specific clinic at a certain time to access the available program, were also excluded.

The studies included in this review were identified through the reference lists of previous reviews of Internet delivered interventions for anxiety, and via Medline searches. Medline was searched from its inception to August 2013, using the following search terms: ("iCBT" OR "Internet treatment" OR "computer-assisted therapy" OR "online therapy" AND "anxiety"); ("iCBT" OR "Internet treatment" OR "computer-assisted therapy" OR "online therapy" AND "PTSD”); (“iCBT" OR "Internet treatment" OR "computer-assisted therapy" OR "online therapy" AND "phobia"); (“iCBT" OR "Internet treatment" OR "computerassisted therapy" OR "online therapy" AND "OCD"). A total of 176 articles were identified ( 87 from Medline searches; 89 from previously published reviews). The titles and abstracts of these articles were inspected independently by two review authors (JS and PR) and 47 full-text articles were retrieved. Following inspection of the full-text articles, a further ten were excluded due to failure to meet the inclusion criteria discussed above. The current review includes 37 randomized controlled trials that examined the efficacy of iCBT programs, as compared with no intervention or active control. An overview of the findings is displayed in Table 1. All studies reported in the table conducted randomized controlled trials of Internet-delivered CBT (including psychoeducation, applied relaxation, and graded exposure) for adults (aged 18 years and over) diagnosed with a range of anxiety disorders.

\section{Efficacy of iCBT for anxiety disorders}

Several trials of iCBT for generalized anxiety disorder, ${ }^{14,19,31,32}$ panic disorder, ${ }^{5,16,33-40}$ and social phobia ${ }^{17,21-23,41-48}$ were identified. Two trials of iCBT for obsessive-compulsive disorder were identified, ${ }^{49,50}$ whilst one trial was identified for illness anxiety disorder, ${ }^{6}$ specific phobia (spiders), ${ }^{51}$ and post-traumatic stress disorder, ${ }^{52}$ respectively. One trial reported the pooled results of separate iCBT programs targeting a range of anxiety disorders (including panic disorder, social phobia, and generalized anxiety disorder) as well as major depression. ${ }^{53}$ Finally, there were four trials that focused on transdiagnostic therapy for either a range of comorbid anxiety disorders, or comorbid anxiety and depression. ${ }^{54-57}$

When compared with waitlist control groups, the findings indicate that $\mathrm{iCBT}$ treatments are effective in reducing symptoms of anxiety in adults with a range of anxiety disorders (24 comparisons; between-group effect size range 0.27-1.47). iCBT was found to be superior to wait list controls in all studies except one..$^{40}$ In the one study reporting equivocal results, adherence was particularly low (only $27 \%$ of the intervention group completed four or more of the six lessons), and reanalysis of the data indicated that there were statistically significant differences between intervention and control groups when only completer data were included. ${ }^{40}$ When compared with attention controls, iCBT was superior in all cases across all the anxiety disorders studied (six comparisons; between-group effect size range 0.38-2.53). iCBT was also comparable with other types of therapy, such as individual face-to-face therapy ${ }^{38,39,48,51}$ (four comparisons; between-group effect size range 0.01-0.19) and Internet-delivered psychodynamic therapy ${ }^{31}$ (betweengroup effect size 0.14 ). When comparing iCBT with faceto-face group therapy, there were no significant differences between the treatment conditions in terms of symptom reduction ${ }^{23,33}$ (two comparisons; between-group effect size range $0.00-0.41$ ).

\section{Internet versus face-to-face delivery of CBT for anxiety disorders}

Preliminary findings indicate that iCBT treatments for anxiety disorders are as efficacious as face-to-face treatments delivered individually ${ }^{38,39,48,51}$ or in a group context. ${ }^{23,33}$ Overall, the results suggest that Internet-administered self-help plus minimal therapist contact via email can be equally effective as traditional individual CBT for both panic disorder and social phobia, whilst involving considerably less clinician time. Treatment outcomes were found to be similar regardless of the modality of treatment delivery, but there were other differences between treatment groups completing face-toface and Internet-delivered CBT that should be noted. In their investigation of treatments for panic disorder, Carlbring et $\mathrm{l}^{38}$ found that when compared with a face-to-face therapy group, the iCBT group was considerably less likely to finish treatment modules in the allocated timeframe and tended to have lower ratings of treatment credibility. Whilst Kiropoulos et al, ${ }^{39}$ also investigating treatments for panic disorder, found no differences in treatment outcomes, credibility, or satisfaction between groups completing face-to-face 


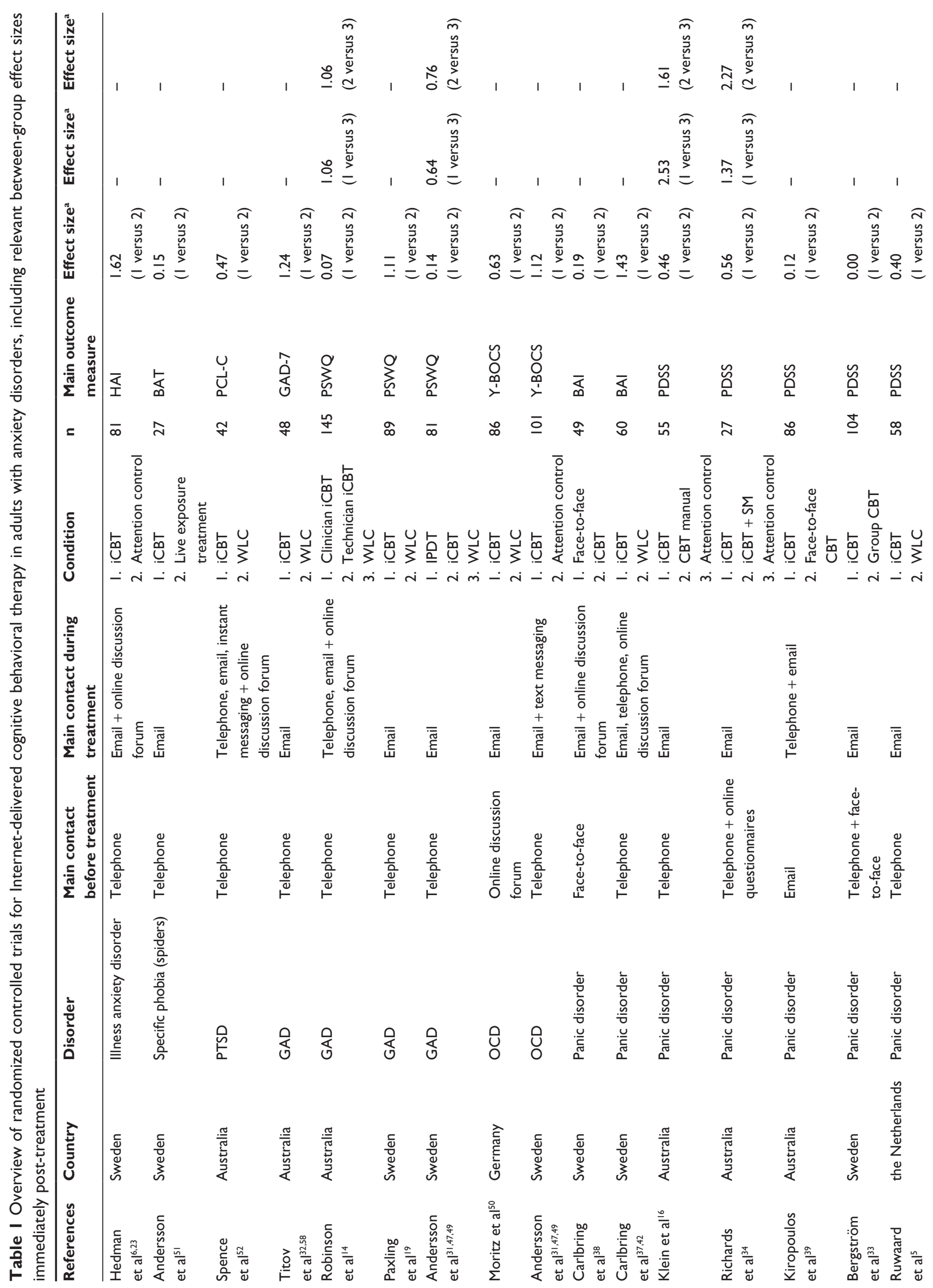




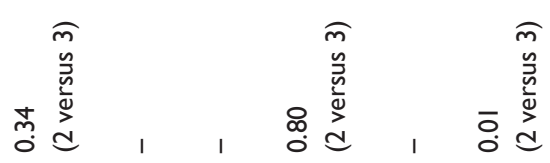

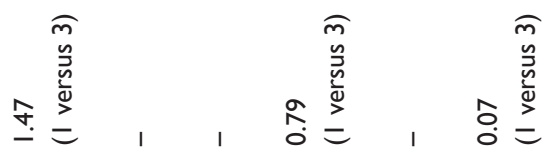

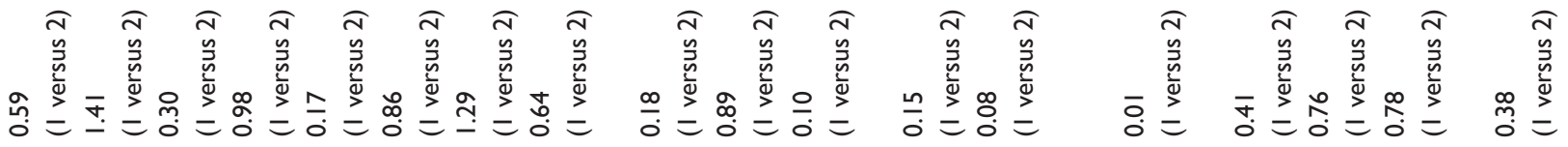

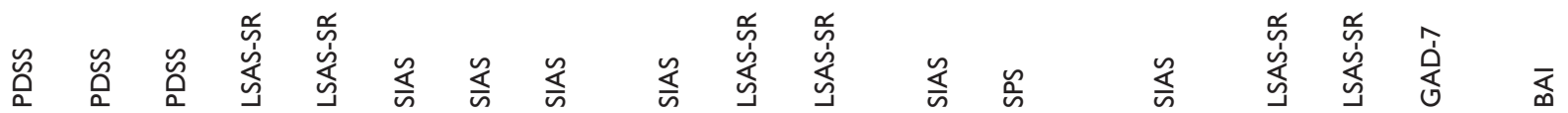

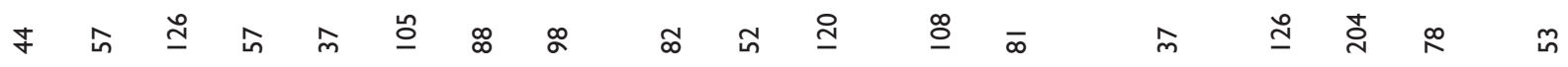

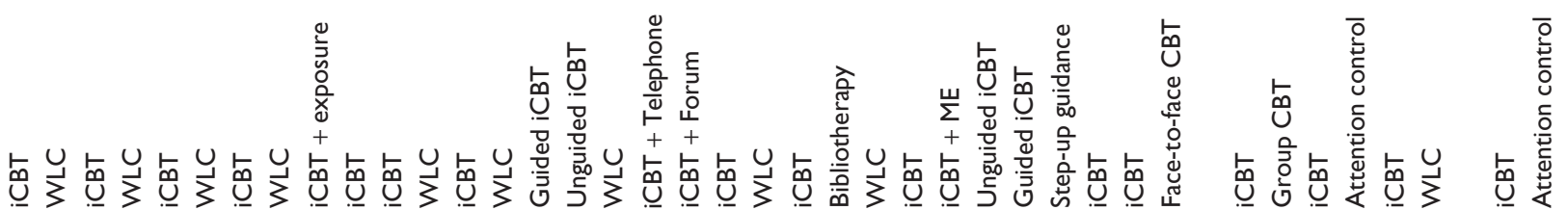

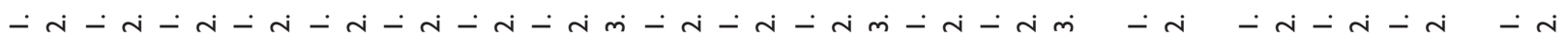

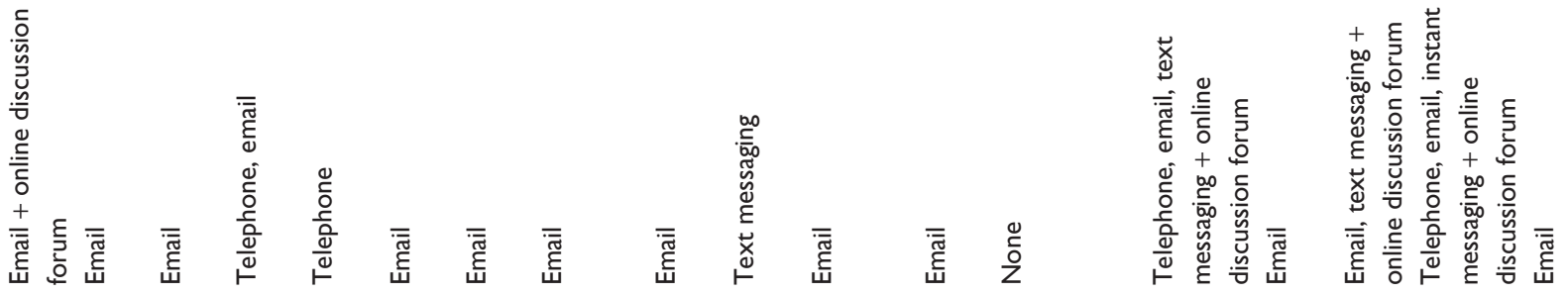

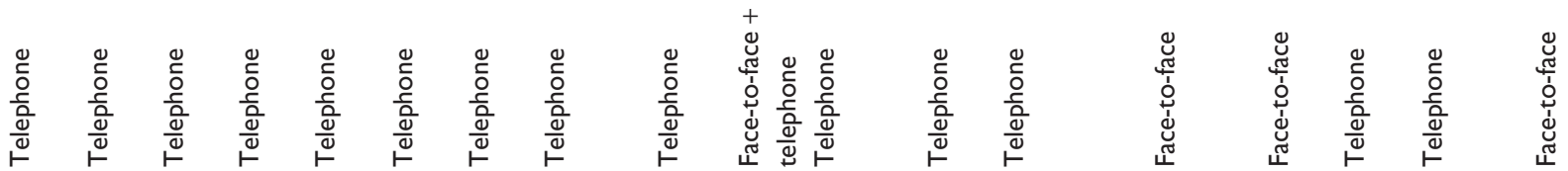

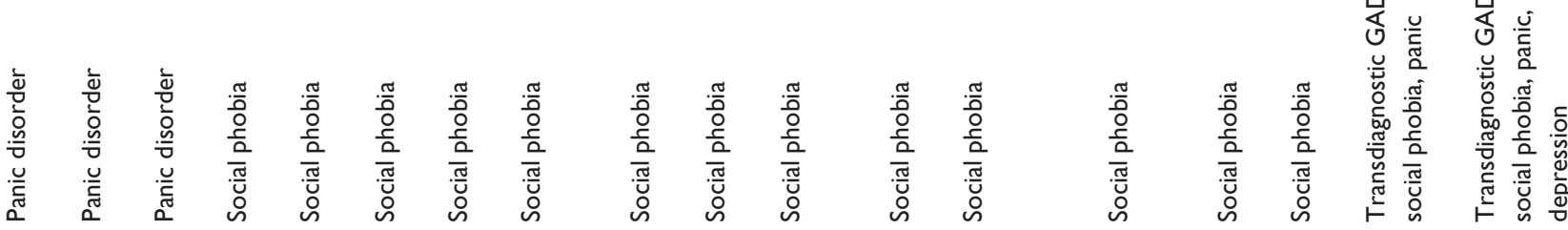

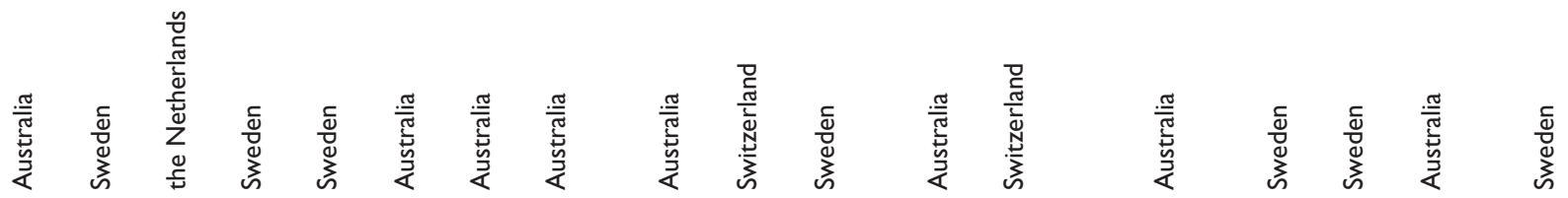

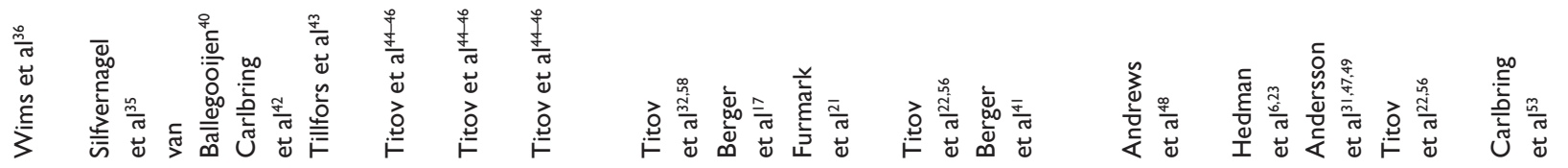




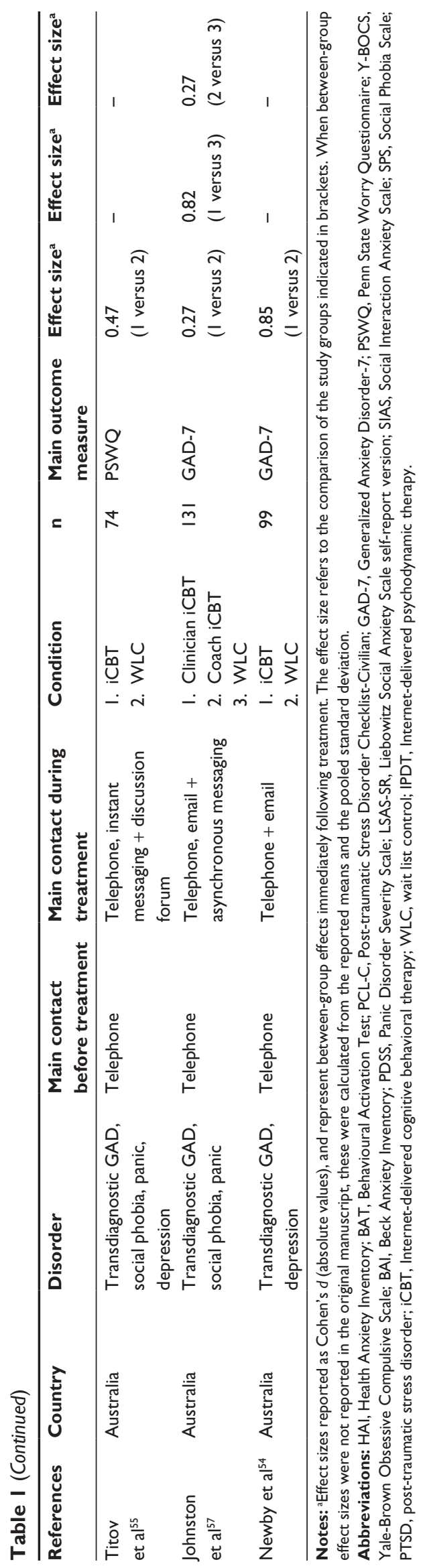

and Internet-delivered CBT, those completing face-to-face therapy were more likely to report enjoyable communications with their therapist. Therapist ratings of treatment compliance and understanding of the CBT materials were also higher in the face-to-face group when compared with those in the iCBT group..$^{39}$ Despite these differences in treatment dosage, credibility, and communication, overall efficacy was found to be equivalent regardless of treatment modality.

One clear advantage of iCBT when compared with traditional face-to-face therapy is the reduction in clinician time. Andrews et al ${ }^{48}$ for example, found that the total amount of clinician time devoted to face-to-face CBT patients was 13 times higher than that required for each iCBT patient. This has clear implications in terms of health care costs, and also allows the clinician to allocate more time to those patients who require more intensive therapy, due to case severity or other clinical complications.

\section{Importance of therapist guidance}

The role and necessity of therapist guidance in iCBT for anxiety disorders is largely unclear. ${ }^{41}$ The amount of therapist support in the included studies varied widely from no support, to small amounts of contact (via telephone or email), to intensive involvement with levels similar to that seen in the face-to-face modalities. In a meta-analysis of iCBT treatments for anxiety and depressive disorders, effect sizes were found to be moderated by the extent of therapist support, and adherence was higher in those interventions that were guided by a clinician. ${ }^{18,51}$ Titov et a $\mathrm{l}^{46}$ compared guided and unguided iCBT for social phobia and found that those in the unguided group were considerably less likely to complete the course (33\% versus $77 \%$ ). Overall, the unguided group benefited less from the treatment (between-group effect size 0.64 ), but in the subgroup of participants who did complete the course, treatment outcomes were similar for those in the guided and unguided groups.

However, there is also evidence to suggest that iCBT can be effective without any therapist guidance. Self-guided iCBT for obsessive-compulsive disorder has been shown to be efficacious without any clinician input, indicating that people with obsessive-compulsive disorder may benefit from highly structured self-guided interventions. ${ }^{50}$ This study did not compare guided and unguided iCBT, so the effect of guidance on treatment efficacy could not be explored. Amongst individuals with social phobia, Berger et $\mathrm{al}^{41}$ compared three iCBT treatment groups that differed only in terms of the amount of guidance received, ie, no guidance, minimal guidance, or flexible guidance, that was responsive to participant 
demand. All three treatment groups demonstrated significant reductions in symptom severity and there were no statistically significant differences between the three conditions in terms of remission from social phobia, clinically significant change, or treatment adherence (between-group effect size 0.08). Participants in the guided treatment groups did, however, report higher treatment satisfaction. In a sample of patients with social phobia, Furmark et $\mathrm{al}^{21}$ compared guided iCBT with unguided bibliotherapy. The bibliotherapy condition consisted of a computerized CBT manual which was adapted for Internet use and included chapters on psychoeducation, cognitive restructuring, exposure, attention training, social skills training, and relapse prevention. In order to be consistent with the guided iCBT course, the chapters were presented as modules to be completed on a weekly basis and were supplemented with homework assignments and additional information. Immediately following treatment, both groups showed similar reductions in symptoms of social phobia (between-group effect size 0.10 ). At one year follow-up, however, the guided iCBT group demonstrated better long-term improvements.

The research thus far indicates that therapist guidance may not be necessary for iCBT to be effective for immediate gains, but may be more important in longer-term maintenance of symptom improvement and maximizing patient adherence. Therapist guidance is associated with monetary costs and is dependent on clinician availability, thereby placing limits on the scalability of iCBT. For the cost-effectiveness and scalability of iCBT to be maximized, further investigations of the optimal amount of therapist guidance needed to generate positive patient outcomes is necessary. Following on from their study of therapist guidance, Berger et $\mathrm{al}^{41}$ suggested a stepped-care approach to therapist guidance in which lowercost pure self-help interventions are the first line of treatment. These unguided programs could then be supplemented with enhanced therapist support for those who fail to benefit from self-guided therapy. Determining the baseline characteristics of those who would benefit from stepped-up therapist support prior to treatment commencement is another fruitful avenue for future research.

\section{Role of therapist experience in iCBT for anxiety disorders}

A few studies have explored the impact of therapist experience on the efficacy of iCBT for anxiety disorders. Andersson et $\mathrm{al}^{47}$ compared experienced clinicians with clinical psychology students and found that therapist experience did not result in different clinical outcomes for patients with social phobia. The less experienced students logged in more frequently than the experienced clinicians, suggesting that they needed more time to support their patients, but clinical outcomes were similar regardless of therapist experience. A series of studies also compared clinician-assisted and technician-assisted iCBT for social phobia and generalized anxiety disorder. ${ }^{14,58}$ Overall, technician-assisted iCBT generated outcomes similar to those of clinician-assisted iCBT, suggesting that clinical experience is not necessary for successful iCBT guidance. The technician in these studies was able to refer patients to a clinician if deemed necessary, but this only occurred in a very small proportion of patients (about 10\%). For iCBT programs that include some level of guidance, nonclinical personnel may be able to provide the bulk of this support, thereby reducing costs and facilitating the efficient use of clinician time.

\section{Factors moderating the efficacy of iCBT for anxiety disorders}

In the studies reviewed, factors associated with treatment efficacy and, relatedly, treatment adherence were rarely identified or discussed. Titov et $\mathrm{al}^{45}$ found that pretreatment expectancies related to changes in anxiety symptoms were moderately correlated with response to iCBT for social phobia. Berger et $\mathrm{l}^{41}$ found that baseline demographic and clinical variables did not predict treatment response in their iCBT program for social phobia, but variables related to program usage (such as number of lessons completed, total time spent in the program, number of mouse clicks in the program, and number of online diary entries) did predict treatment outcomes. These studies suggest that patient engagement and expectancies may be key factors in moderating the efficacy of iCBT for social phobia. With regard to iCBT for panic disorder, Ruwaard et $\mathrm{al}^{5}$ found that female sex, higher pretreatment symptom levels, and a higher frequency of panic attacks at baseline were associated with poorer treatment response. Silfvernagel et a ${ }^{35}$ were specifically interested in the effect of age on treatment response to iCBT for panic disorder, but found that age did not moderate treatment outcomes. Poorer adherence to iCBT, on the other hand, has been found to be related to more severe baseline symptoms and higher baseline psychological distress, ${ }^{14,45}$ as well as less therapist involvement in terms of telephone contact. ${ }^{37}$ In terms of both efficacy and adherence, these findings suggest that iCBT may not be suitable for more severe cases and that patient engagement and expectancies may also be important factors. Future research should focus on clarifying the factors which may moderate the efficacy of iCBT for anxiety 
disorders, so that evidence-based contraindications for iCBT can be formulated, and patients directed to the most appropriate modality of care before commencement of treatment.

\section{Enhancements to iCBT for anxiety disorders}

Although Internet-based treatments are effective in the treatment of anxiety disorders, a number of studies have also investigated the inclusion of enhancements additional to the standard iCBT content. Richards et $\mathrm{al}^{34}$ found that adding a stress management component to iCBT for panic disorder produced better clinical outcomes immediately post-treatment when compared with iCBT alone, but these differences were no longer apparent at longer-term follow-up. Titov et $\mathrm{al}^{22}$ added self-guided motivational enhancement strategies to iCBT for social phobia, but found that addition of motivational techniques did not improve clinical outcomes when compared with the standard iCBT program. The motivational group, however, had increased levels of adherence when compared with the standard iCBT group. Tillfors et $\mathrm{al}^{43}$ explored the addition of face-to-face exposure sessions to the standard iCBT content for social phobia and found that the supplementary exposure had no impact on treatment efficacy. Whilst the literature is sparse, there is currently no evidence to suggest that enhancing standard iCBT content with additional clinical elements leads to better patient outcomes.

\section{Transdiagnostic approaches to iCBT: issue of comorbidity}

Anxiety disorders frequently co-occur with each other, and are often comorbid with major depression. Similar cognitive, behavioral, and emotional processes maintain both anxiety and affective disorders, and as a result, iCBT programs for individual disorders share many common elements. Recently, researchers have focused on these common elements and developed iCBT programs that target a range of psychiatric disorders. Carlbring et $\mathrm{l}^{53}$ dismantled their iCBT courses for generalized anxiety disorder, panic disorder, social phobia, and depression into 16 discrete modules which could then be prescribed to patients depending on their presenting symptoms. Each patient therefore received an individualized program of 6-10 modules, the structure and content of which was determined on the basis of clinician judgment, structured diagnostic interview, and patient preference. A similar procedure was also used by the same research group in a sample of individuals experiencing panic attacks. ${ }^{35}$ In both studies, participants in the treatment groups demonstrated better patient outcomes than those in the control groups, and these effects were sustained over longer-term follow-up.

Another approach has been to develop transdiagnostic programs specifically designed for patients presenting with psychiatric comorbidity. Titov et $\mathrm{al}^{56}$ developed a transdiagnostic iCBT program for anxiety disorders, in which elements from existing disorder-specific programs for generalized anxiety disorder, panic disorder, and social phobia were combined into a single six-lesson iCBT course. Relative to control, individuals in the transdiagnostic treatment group reported superior outcomes in terms of disorder-specific measures, as well as measures of common factors such as neuroticism, indicating generalized benefits across the psychiatric spectrum. Adherence and treatment satisfaction were also high. These findings were replicated by Johnston et al. ${ }^{57}$ Titov et $\mathrm{al}^{55}$ and Newby et $\mathrm{al}^{54}$ have also developed transdiagnostic programs for comorbid anxiety and depression and similarly found that, relative to control, the treatment group demonstrated superior reductions in disorder-specific outcomes, as well as more generalized factors such as neuroticism.

\section{Limitations of current review}

The current review needs to be considered within the context of some limitations. A formal methodological quality evaluation was not undertaken, but there are methodological problems observed in the primary studies that limit the conclusions of our review. For example, sample sizes in the primary studies were frequently small, and the representativeness of the study participants was often limited. The review covers a range of anxiety and related disorders, which required diverse interventions and clinical methods, whilst treatment outcomes were established through a variety of methods. The lack of standardized treatments, techniques, and assessments further limit the conclusions of this review.

\section{Future challenges}

iCBT for anxiety disorders is efficacious, cost-effective, and scalable, and an important treatment alternative to faceto-face CBT. As such, current research is focusing on the effectiveness of these treatments outside of highly controlled research settings, with preliminary results indicating that iCBT is effective when disseminated into primary care. ${ }^{54,59-62}$ Future research needs to focus on the optimal level of guidance required to generate maximum patient benefits, whilst balancing the efficient use of clinician time and resources. Evidence-based contraindications to iCBT should also be developed so that the choice of treatment modality accurately reflects patients' needs. Further research should be conducted 
into the effective elements of iCBT, as well as the extent to which therapy enhancers and advancing technology can be accommodated into established iCBT frameworks. Internet interventions will likely follow the move towards mobile Internet access, building upon mobile telephone platforms which will ultimately allow greater patient access. ${ }^{63}$ iCBT for depression has recently been successfully adapted for a mobile telephone platform, ${ }^{64}$ and similar advances in anxiety disorder treatment should follow in this vein. The results considered in this review are mostly from Australia and Sweden, along with a handful of other affluent, first-world countries from north-western Europe. These countries represent some of strongest world economies, and tend to work within socialized health care models. The sample group is therefore unlikely to be representative of global populations, and future research should focus on the extent to which technology-assisted interventions can generate positive outcomes in the developing world.

\section{Disclosure}

The authors report no conflicts of interest in this work.

\section{References}

1. Slade T, Johnston A, Browne MAO, Andrews G, Whiteford H. 2007 National Survey of Mental Health and Wellbeing: methods and key findings. Aust N Z J Psychiatry. 2009;43(7):594-605.

2. Burgess PM, Pirkis JE, Slade TN, et al. Service use for mental health problems: findings from the 2007 National Survey of Mental Health and Wellbeing. Aust N Z J Psychiatry. 2009;43(7):615-623.

3. American Psychiatric Association. Diagnostic and Statistical Manual of Mental Disorders, Fifth Edition (DSM-5). Washington, DC, USA: American Psychiatric Association; 2013.

4. Andrews G, Sanderson K, Slade T, Issakidis C. Why does the burden of disease persist? Relating the burden of anxiety and depression to effectiveness of treatment. Bull World Health Organ. 2000;78(4): 446-454.

5. Ruwaard J, Broeksteeg J, Schrieken B, Emmelkamp P, Lange A. Web-based therapist-assisted cognitive behavioral treatment of panic symptoms: A randomized controlled trial with a three-year follow-up. J Anxiety Disord. 2010;24(4):387-396.

6. Hedman E, Andersson G, Andersson E, et al. Internet-based cognitivebehavioural therapy for severe health anxiety: randomised controlled trial. Br J Psychiatry. 2011;198(3):230-236.

7. American Psychiatric Association. Diagnostic and Statistical Manual of Mental Disorders, Fourth Edition, Text Revision (DSM-IV-TR). Washington, DC, USA: American Psychiatric Association; 1994.

8. Butler AC, Chapman JE, Forman EM, Beck AT. The empirical status of cognitive-behavioral therapy: a review of meta-analyses. Clin Psychol Rev. 2006;26(1):17-31.

9. Baldwin DS, Anderson IM, Nutt DJ, et al. Evidence-based guidelines for the pharmacological treatment of anxiety disorders: recommendations from the British Association for Psychopharmacology. J Psychopharmacol (Oxf). 2005;19(6):567-596.

10. Mitte K. Meta-analysis of cognitive-behavioral treatments for generalized anxiety disorder: a comparison with pharmacotherapy. Psychol Bull. 2005;131(5):785-795.

11. Taylor CB, Chang VY. Issues in the dissemination of cognitive-behavior therapy. Nord J Psychiatry. 2008;62(S47):37-44.
12. Johansson R, Andersson G. Internet-based psychological treatments for depression. Expert Rev Neurother. 2012;12(7):861-870.

13. Acarturk C, Cuijpers P, Van Straten A, De Graaf R. Psychological treatment of social anxiety disorder: a meta-analysis. Psychol Med. 2009;39(2):241-254.

14. Robinson E, Titov N, Andrews G, et al. Internet treatment for generalized anxiety disorder: a randomized controlled trial comparing clinician vs technician assistance. PLoS One. 2010;5(6):e10942.

15. Andrews G, Cuijpers P, Craske MG, McEvoy P, Titov N. Computer therapy for the anxiety and depressive disorders is effective, acceptable and practical health care: A meta-analysis. PLoS One. 2010;5(10):e13196.

16. Klein B, Richards JC, Austin DW. Efficacy of Internet therapy for panic disorder. J Behav Ther Exp Psychiatry. 2006;37(3):213-238.

17. Berger T, Hohl E, Caspar F. Internet-based treatment for social phobia: a randomized controlled trial. J Clin Psychol. 2009;65(10): $1021-1035$.

18. Spek V, Cuijpers P, Nyklícek I, et al. Internet-based cognitive behaviour therapy for symptoms of depression and anxiety: a meta-analysis. Psychol Med. 2007;37(3):319-328.

19. Paxling B, Almlöv J, Dahlin M, et al. Guided Internet-delivered cognitive behavior therapy for generalized anxiety disorder: a randomized controlled trial. Cogn Behav Ther. 2011;40(3):159-173.

20. Dear BF, Titov N, Schwencke G, et al. An open trial of a brief transdiagnostic Internet treatment for anxiety and depression. Behav Res Ther. 2011;49(12):830-837.

21. Furmark T, Carlbring P, Hedman E, et al. Guided and unguided self-help for social anxiety disorder: randomised controlled trial. Br J Psychiatry. 2009;195(5):440-447.

22. Titov N, Andrews G, Schwencke G, et al. Randomized controlled trial of Internet cognitive behavioural treatment for social phobia with and without motivational enhancement strategies. Aust N Z J Psychiatry. 2010;44(10):938-945.

23. Hedman E, Andersson G, Ljótsson B, et al. Internet-based cognitive behavior therapy vs cognitive behavioral group therapy for social anxiety disorder: a randomized controlled non-inferiority trial. PLoS One. 2011;6(3):e18001.

24. Cuijpers P, Marks IM, van Straten A, et al. Computer-aided psychotherapy for anxiety disorders: a meta-analytic review. Cogn Behav Ther. 2009;38(2):66-82.

25. Reger MA, Gahm GA. A meta-analysis of the effects of Internet and computer based cognitive behavioral treatments for anxiety. $J$ Clin Psychol. 2009;65(1):53-75.

26. Andersson G, Cuijpers P, Carlbring P, Lindefors N. Effects of Internetdelivered cognitive behaviour therapy for anxiety and mood disorders. Psychiatry. 2007;2:9-14.

27. Christensen H, Griffiths KM, Farrer L. Adherence in Internet interventions for anxiety and depression: systematic review. $J$ Med Internet Res. 2009;11(2).

28. Newman MG, Szkodny LE, Llera SJ, Przeworski A. A review of technology-assisted self-help and minimal contact therapies for anxiety and depression: is human contact necessary for therapeutic efficacy? Clin Psychol Rev. 2011;31(1):89-103.

29. Griffiths KM, Farrer L, Christensen H. The efficacy of Internet interventions for depression and anxiety disorders: a review of randomised controlled trials. Med J Aust. 2010;192(11):S4-S11.

30. Cuijpers P, Donker T, Van Straten A, Li J, Andersson G. Is guided self-help as effective as face-to-face psychotherapy for depression and anxiety disorders? A systematic review and meta-analysis of comparative outcome studies. Psychol Med. 2010;40(12):1943-1957.

31. Andersson G, Paxling B, Roch-Norlund P, et al. Internet-based psychodynamic versus cognitive behavioral guided self-help for generalized anxiety disorder: a randomized controlled trial. Psychother Psychosom. 2012;81(6):344-355.

32. Titov N, Andrews G, Robinson E, et al. Clinician-assisted Internet-based treatment is effective for generalized anxiety disorder: randomized controlled trial. Aust N Z J Psychiatry. 2009;43(10):905-912. 
33. Bergström J, Andersson G, Ljótsson B, et al. Internet-versus groupadministered cognitive behaviour therapy for panic disorder in a psychiatric setting: a randomised trial. BMC Psychiatry. 2010;10:54.

34. Richards JC, Klein B, Austin DW. Internet cognitive behavioural therapy for panic disorder: Does the inclusion of stress management information improve end-state functioning? Clin Psychol. 2006;10(1):2-15.

35. Silfvernagel K, Carlbring $\mathrm{P}, \mathrm{Kabo} \mathrm{J}$, et al. Individually tailored Internet-based treatment for young adults and adults with panic attacks: randomized controlled trial. J Med Internet Res. 2012;14(3).

36. Wims E, Titov N, Andrews G, Choi I. Clinician-assisted Internet-based treatment is effective for panic: a randomized controlled trial. Aust N Z J Psychiatry. 2010;44(7):599-607.

37. Carlbring $\mathrm{P}$, Bohman S, Brunt S, et al. Remote treatment of panic disorder: a randomized trial of Internet-based cognitive behavior therapy supplemented with telephone calls. Am J Psychiatry. 2006;163(12): 2119-2125.

38. Carlbring P, Nilsson-Ihrfelt E, Waara J, et al. Treatment of panic disorder: live therapy vs self-help via the Internet. Behav Res Ther. 2005;43(10):1321-1333.

39. Kiropoulos LA, Klein B, Austin DW, et al. Is Internet-based CBT for panic disorder and agoraphobia as effective as face-to-face CBT? J Anxiety Disord. 2008;22(8):1273-1284.

40. van Ballegooijen W, Riper H, Klein B, et al. An Internet-based guided self-help intervention for panic symptoms: randomized controlled trial. J Med Internet Res. 2013;15(7).

41. Berger T, Caspar F, Richardson R, et al. Internet-based treatment of social phobia: a randomized controlled trial comparing unguided with two types of guided self-help. Behav Res Ther. 2011;49(3):158-169.

42. Carlbring P, Gunnarsdóttir M, Hedensjö L, et al. Treatment of social phobia: randomised trial of Internet-delivered cognitive-behavioural therapy with telephone support. Br J Psychiatry. 2007;190(2):123-128.

43. Tillfors M, Carlbring P, Furmark T, et al. Treating university students with social phobia and public speaking fears: Internet delivered selfhelp with or without live group exposure sessions. Depress Anxiety. 2008;25(8):708-717.

44. Titov N, Andrews G, Schwencke G, Drobny J, Einstein D. Shyness 1: distance treatment of social phobia over the Internet. Aust N Z J Psychiatry. 2008;42(7):585-594.

45. Titov N, Andrews G, Schwencke G. Shyness 2: treating social phobia online: replication and extension. Aust $N$ Z J Psychiatry. 2008;42(7):595-605.

46. Titov N, Andrews G, Choi I, Schwencke G, Mahoney A. Shyness 3: randomized controlled trial of guided versus unguided Internet-based CBT for social phobia. Aust N Z J Psychiatry. 2008;42(12):1030-1040.

47. Andersson G, Carlbring P, Furmark T. Therapist experience and knowledge acquisition in Internet-delivered CBT for social anxiety disorder: a randomized controlled trial. PLoS One. 2012;7(5):e37411.

48. Andrews G, Davies M, Titov N. Effectiveness randomized controlled trial of face-to-face versus Internet cognitive behaviour therapy for social phobia. Aust N Z J Psychiatry. 2011;45(4):337-340.

49. Andersson E, Enander J, Andrén P, et al. Internet-based cognitive behaviour therapy for obsessive-compulsive disorder: a randomized controlled trial. Psychol Med. 2012;21:1-11.
50. Moritz S, Jelinek L, Hauschildt M, Naber D. How to treat the untreated: effectiveness of a self-help metacognitive training program (myMCT) for obsessive-compulsive disorder. Dialogues Clin Neurosci. 2010;12(2):209-220.

51. Andersson G, Waara J, Jonsson U, et al. Internet-based self help versus one session exposure in the treatment of spider phobia: a randomized controlled trial. Cogn Behav Ther. 2009;38(2):114-120.

52. Spence J, Titov N, Dear BF, et al. Randomized controlled trial of Internetdelivered cognitive behavioral therapy for post-traumatic stress disorder. Macquarie University Research Online. 2011; 28(7):541-550.

53. Carlbring P, Maurin L, Törngren C, et al. Individually-tailored, Internet-based treatment for anxiety disorders: a randomized controlled trial. Behav Res Ther. 2011;49(1):18-24.

54. Newby J, Mackenzie A, Williams A, et al. Internet cognitive behavioural therapy for mixed anxiety and depression: a randomized controlled trial and evidence of effectiveness in primary care. Psychol Med. 2013:1-14.

55. Titov N, Dear BF, Schwencke G, et al. Transdiagnostic Internet treatment for anxiety and depression: a randomised controlled trial. Behav Res Ther. 2011;49(8):441-452.

56. Titov N, Andrews G, Johnston L, Robinson E, Spence J. Transdiagnostic Internet treatment for anxiety disorders: a randomized controlled trial. Behav Res Ther. 2010;48(9):890-899.

57. Johnston L, Titov N, Andrews G, Spence J, Dear BF. A RCT of a transdiagnostic Internet-delivered treatment for three anxiety disorders: examination of support roles and disorder-specific outcomes. PLoS One. 2011;6(11):e28079.

58. Titov N, Andrews G, Schwencke G, et al. An RCT comparing effect of two types of support on severity of symptoms for people completing Internet-based cognitive behaviour therapy for social phobia. Aust NZJ Psychiatry. 2009;43(10):920-926.

59. Mewton L, Wong N, Andrews G. The effectiveness of Internet cognitive behavioural therapy for generalized anxiety disorder in clinical practice. Depress Anxiety. 2012;59(10):843-849.

60. Hilvert-Bruce Z, Rossouw PJ, Wong N, Sunderland M, Andrews G. Adherence as a determinant of effectiveness of Internet cognitive behavioural therapy for anxiety and depressive disorders. Behav Res Ther. 2012;50(7-8):463-468.

61. Sunderland M, Wong N, Hilvert-Bruce Z, Andrews G. Investigating trajectories of change in psychological distress amongst patients with depression and generalised anxiety disorder treated with Internet cognitive behavioural therapy. Behav Res Ther. 2012;50(6): 374-380

62. Williams AD, Andrews G. The effectiveness of Internet cognitive behavioural therapy (iCBT) for depression in primary care: a quality assurance study. PLoS One. 2013;8(2):e57447.

63. Mohr DC, Siddique J, Ho J, Duffecy J, Jin L. Interest in behavioral and psychological treatments delivered face-to-face, by telephone, and by Internet. Ann Behav Med. 2010;40(1):89-98.

64. Watts S, Newby J, Mewton L, Andrews G. A clinical audit of changes in suicide ideas with Internet treatment for depression. BMJ Open. 2012;2:e001558.
Psychology Research and Behavior Management

\section{Publish your work in this journal}

Psychology Research and Behavior Management is an international, peerreviewed, open access journal focusing on the science of psychology and its application in behavior management to develop improved outcomes in the clinical, educational, sports and business arenas. Specific topics covered include: Neuroscience, memory \& decision making; Behavior

\section{Dovepress}

modification \& management; Clinical applications; Business \& sports performance management; Social and developmental studies; Animal studies. The manuscript management system is completely online and includes a quick and fair peer-review system. Visit http://www.dovepress. com/testimonials.php to read real quotes from published authors. 